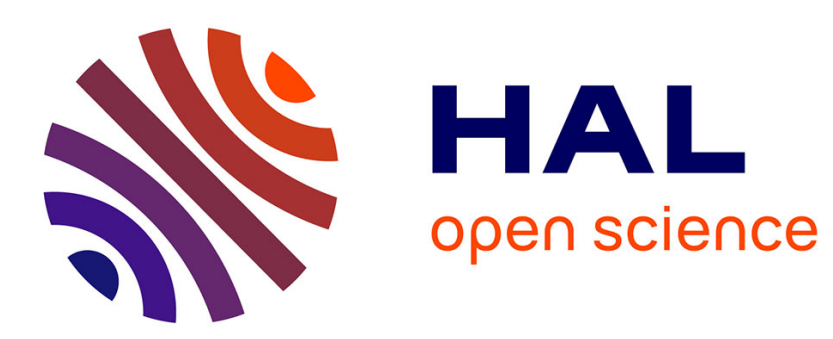

\title{
A unique robust controller for tracking and stabilisation of non-holonomic vehicles
}

\author{
Mohamed Maghenem, Antonio Loria, Elena Panteley
}

\section{To cite this version:}

Mohamed Maghenem, Antonio Loria, Elena Panteley. A unique robust controller for tracking and stabilisation of non-holonomic vehicles. International Journal of Control, 2020, 93 (10), pp.2302-2313. 10.1080/00207179.2018.1554270 . hal-02367651

\section{HAL Id: hal-02367651 https://hal.science/hal-02367651}

Submitted on 5 Mar 2020

HAL is a multi-disciplinary open access archive for the deposit and dissemination of scientific research documents, whether they are published or not. The documents may come from teaching and research institutions in France or abroad, or from public or private research centers.
L'archive ouverte pluridisciplinaire HAL, est destinée au dépôt et à la diffusion de documents scientifiques de niveau recherche, publiés ou non, émanant des établissements d'enseignement et de recherche français ou étrangers, des laboratoires publics ou privés. 


\title{
A unique robust controller for tracking and stabilization of nonholonomic vehicles
}

\author{
Mohamed Maghenem $^{\mathrm{a}}$ Antonio Loría ${ }^{\mathrm{b} *}$ Elena Panteley ${ }^{\mathrm{b}, \mathrm{c} \dagger}$ \\ anniversity of California, Santa Cruz, CA, USA; \\ ${ }^{\mathrm{b}}$ CNRS, L2S-Supelec, 3 Rue Joliot Curie, Gif sur Yvette France; \\ 'ITMO University, Kronverkskiy av. 49, Saint Petersburg, 197101, Russia.
}

\section{ARTICLE HISTORY}

\begin{abstract}
After (Lizárraga, 2004) it is known that for nonholonomic systems it is impossible to design a universal controller able to asymptotically stabilize any feasible reference trajectory. In this paper we present a smooth time-varying controller able to stabilize a wide class of reference trajectories that include converging (parking problem) and persistently exciting (tracking problem) ones, as well as set-points. To the best of our knowledge, for the first time in the literature, we establish uniform global asymptotic stability for the origin of the closed-loop system in the kinematics state space. We also show that the kinematics controller renders the system robust to perturbations in the sense of integral-input-to-state stability. Then, we show that for the case in which the velocity dynamics equations are also considered (full model), any velocity-tracking controller with the property that the error velocities are square integrable may be used to ensure global tracking or stabilization. This modularity and robustness of our controller, added to the strength of our stability statements, renders possible the extension of our main results to the difficult scenario of control under parametric uncertainty.
\end{abstract}

\section{KEYWORDS}

Persistency of excitation, autonomous vehicles, Lyapunov's method, input-to-state stability

\section{Introduction}

In the well-known paper Brockett (1983) necessary conditions for asymptotic set-point stabilization of nonlinear systems via continuous controllers are given. In particular, it is showed that nonholonomic systems are not stabilizable to a point via continuous autonomous feedback. The seminal paper Lizárraga (2004) extends the results of Brockett (1983) for smooth control systems by establishing sufficient conditions for the non existence of universal continuous stabilizers (even time-varying) of arbitrary feasible trajectories. A particular but fundamental contribution of Lizárraga (2004) is that for nonholonomic systems it is beyond reach to design a universal controller (even time-varying) capable of stabilizing an arbitrary feasible trajectory.

The work of Mohamed Maghenem was carried out while he was with Univ Paris-Saclay, Saclay, France.

*Corresponding author. Email: loria@lss.supelec.fr

$\dagger$ The work of E. Panteley is partially suported by the Government of the Russian Federation under grant 074-U01 
The results of Brockett (1983) certainly triggered the interest of the research community on different stabilization and tracking control problems for nonholonomic systems and, in accordance with Lizárraga (2004), available results apply either to the set-point control problem — see e.g. Astolfi (1996); Bayat, Mobayen, and Javadi (2016), to the so-called parking control problem - see e.g. Lee, Song, Lee, and Teng (2001); Morin and Samson (2003), or to other kinds of restricted time-varying trajectories see e.g. Dixon, Dawson, Zergeroglu, and Behal (2001); Kanayama, Kimura, Miyazaki, and Naguchi (1990); Loría, Dasdemir, and Alvarez-Jarquin (2016). Tracking control in the sense defined in Kanayama et al. (1990) is addressed, e.g., via nonlinear backstepping control in Jiang and Nijmeijer (1997). There also exist several remarkable works under stringent conditions: for instance, parametric uncertainty is coped with via adaptive control in Dixon, de Queiroz, Dawson, and Flynn (2004); Fukao, Nakagawa, and Adachi (2000); Huang, Wen, Wang, and Jiang (2014), control under input constraints is addressed e.g. in Consolini, Morbidi, Prattichizzo, and Tosques (2008); Jiang, Lefeber, and Nijmeijer (2001); Lee et al. (2001).

In Panteley, Lefeber, Loría, and Nijmeijer (1998) a simple linear time-varying controller was proposed and, for the first time in the literature, persistency of excitation was explicitly imposed as a necessary and sufficient condition for stabilization of a time-varying trajectory. The underlying ideas, however, are already present in early work by C. Samson de Wit, Khennouf, Samson, and Sørdalen (1993). Ever since, persistency of excitation has been recurrently used in the literature to design smooth controllers for autonomous vehicles - see e.g., Dixon, Dawson, Zhang, and Zergeroglu (2000); Lefeber (2000); Miao and Wang (2015); Wang, Miao, Zhong, and Pan (2015) to mention a few. However, controllers relying on persistency-of-excitation conditions on the reference trajectories fail in other stabilization tasks, as for instance, in the case that the reference velocities tend to zero, as in the case of the parking-control problem Lee et al. (2001). In the latter, as also in Cao and Tian (2007), nonlinear time-varying controllers are designed to allow for reference velocity trajectories that converge to zero. Furthermore, it is worth to emphasize that Lee et al. (2001) covers the case when both the angular and forward velocity may converge to zero.

For the set-point stabilization problem it is also possible to use persistency of excitation as a stabilization mechanism, but the property needs to be redefined for functions that are state-dependent. To the best of our knowledge, this was done for the first time in Loría, Panteley, and Teel (1999) where, inspired by the seminal paper Samson (1995), the so-called $\delta$-persistently-exciting controllers were introduced ${ }^{1}$. See also Wang et al. (2015) for more recent references.

Designing a unique controller capable of stabilizing bounded converging or diverging (i.e., persistently exciting), reference velocity trajectories is not only a major challenge but, based on Lizárraga (2004), it may not appear as an overstatement to say that it is close to the broadest solvable control problem for nonholonomic systems. To the best of our knowledge, it has been addressed only in Dixon et al. (2004); Do, Jiang, and Pan (2004b); Lee et al. (2001); Miao and Wang (2015); Morin and Samson (2003); Wang et al. (2015).

The problem is solved for particular cases of the reference velocity trajectories in Lee et al. (2001), e.g., it is required that either the forward or the angular reference velocities is separated from zero. In Morin and Samson (2003) the elegant transversefunctions approach is presented; a unified velocity controller for generic chained-form

${ }^{1}$ Persistency of excitation in the sense defined in Loría et al. (1999) is also implicitly used in the earlier reference Samson (1993) 
systems (hence beyond the unicycle particular system) is proposed. A similar result is presented in Dixon et al. (2004) using an adaptive-control approach. In the latter two references convergence of the tracking errors in a practical sense is established. The method of Wang et al. (2015), which is based on the design of $\delta$-persistently exciting controllers, is appealing in the sense that it consists in using the combination of a tracking controller and a stabilization controller carefully weighted by a function that depends on the leader velocities, thereby favoring the action of either controller; a similar controller, assuming parametric uncertainty, is presented in Miao and Wang (2015). In Do et al. (2004b) a Lyapunov-based unified controller is proposed in order to make the tracking error converge to zero in either of the tracking and the parking scenarios.

In this paper we address the simultaneous tracking-stabilization problem, based on the approach of Wang et al. (2015). To the best of our knowledge for the first time in the literature, we establish uniform global asymptotic stability (UGAS), as opposed to the weaker property of non-uniform convergence. The importance of UGAS for timevarying systems cannot be overestimated; only this property guarantees robustness of the system with respect to bounded disturbances in the sense of total stability Malkin (1944) — a concept better known nowadays as local input-to-state stability.

Furthermore, our proofs of UGAS and iISS rely on Lyapunov's direct method, which is fundamental to establish asymptotic tracking in the case when the full model (comprising the dynamics and the kinematics equations) is considered. Hence, another contribution of this paper is to establish that any velocity controller that guarantees velocity tracking, including under parametric uncertainty, may be easily incorporated. Such a statement is not possible to obtain without guaranteeing uniform global asymptotic stability for the kinematics model.

The design of our Lyapunov functions follows the efficient Mazenc construction method Mazenc (2003) which, loosely speaking, consists in designing a strict Lyapunov function upon a preliminary non-strict one - see Malisoff and Mazenc (2009) for further detail. For the robustness properties that we establish we also appeal to technical results in Angeli, Ingalls, Sontag, and Wang (2004); Angeli, Sontag, and Wang (2000). Although Lyapunov's first method has been used, e.g., in Do et al. (2004b) the conditions in the latter reference are more conservative.

The rest of the paper is organized as follows. In Section 2 we formulate the control problem and we present our main theoretical findings. In Section 3 we present the proofs of our main results. Simulations that illustrate our theoretical findings are presented in Section 4 and concluding remarks are given in Section 5.

\section{Problem formulation and its solution}

\subsection{Problem statement}

Let us consider the following dynamical model of a force-controlled nonholonomic vehicle:

$$
\begin{aligned}
& \left\{\begin{array}{l}
\dot{x}=v \cos \theta \\
\dot{y}=v \sin \theta \\
\dot{\theta}=\omega
\end{array}\right. \\
& \begin{cases}\dot{v} & =f_{1}(t, v, \omega, z)+g_{1}(t, v, \omega, z) u_{1} \\
\dot{\omega} & =f_{2}(t, v, \omega, z)+g_{2}(t, v, \omega, z) u_{2}\end{cases}
\end{aligned}
$$


where $v$ and $\omega$ denote the forward and angular velocities respectively, the first two elements of $z:=\left[\begin{array}{lll}x & y & \theta\end{array}\right]^{\top}$ correspond to the Cartesian coordinates of a point on the robot with respect to a fixed reference frame, and $\theta$ denotes the robot's orientation with respect to the same frame. The functions $f_{1}$ and $f_{2}$ are assumed to possess the minimal properties for existence and uniqueness of (Caratheodory) solutions, and $u_{1}$ and $u_{2}$ correspond to the two control inputs (proportional to wheel torques). Thus, the equations (1) correspond to the kinematics model while (2) correspond to the force-balance equations.

The tracking-control problem consists in making the robot follow a fictitious reference vehicle modeled by

$$
\begin{aligned}
\dot{x}_{r} & =v_{r} \cos \theta_{r} \\
\dot{y}_{r} & =v_{r} \sin \theta_{r} \\
\dot{\theta}_{r} & =\omega_{r},
\end{aligned}
$$

that moves about with reference velocities $v_{r}(t)$ and $\omega_{r}(t)$. More precisely, it is desired to steer the differences between the Cartesian coordinates to some values $d_{x}, d_{y}$, and to zero the orientation angles and the velocities of the two robots, that is, the quantities

$$
p_{\theta}:=\theta_{r}-\theta, \quad p_{x}:=x_{r}-x-d_{x}, \quad p_{y}:=y_{r}-y-d_{y} .
$$

The distances $d_{x}, d_{y}$ define the position of the robot with respect to the (virtual) leader and are assumed to be constant. Then, as it is customary, we transform the error coordinates $\left[p_{\theta} p_{x} p_{y}\right]$ of the leader robot from the global coordinate frame to local coordinates fixed on the robot, that is, we define

$$
\left[\begin{array}{l}
e_{\theta} \\
e_{x} \\
e_{y}
\end{array}\right]:=\left[\begin{array}{ccc}
1 & 0 & 0 \\
0 & \cos \theta & \sin \theta \\
0 & -\sin \theta & \cos \theta
\end{array}\right]\left[\begin{array}{l}
p_{\theta} \\
p_{x} \\
p_{y}
\end{array}\right]
$$

In these new coordinates, the error kinematics equations become

$$
\begin{aligned}
\dot{e}_{\theta} & =\omega_{r}(t)-\omega \\
\dot{e}_{x} & =\omega e_{y}-v+v_{r}(t) \cos \left(e_{\theta}\right) \\
\dot{e}_{y} & =-\omega e_{x}+v_{r}(t) \sin \left(e_{\theta}\right) .
\end{aligned}
$$

The complete system also includes Eqs (2).

Generally speaking, the control problem consists in steering the error trajectories $e(t)$, which are solutions of (5), to zero via the inputs $u_{1}$ and $u_{2}$ in (2). A natural method consists in designing, first, virtual control laws $w^{*}$ and $v^{*}$ so that,

$$
\lim _{t \rightarrow \infty} e(t)=0, \quad e=\left[\begin{array}{lll}
e_{\theta} & e_{x} & e_{y}
\end{array}\right]^{\top} .
$$

Then, to design control inputs $u_{1}$ and $u_{2}$ such that

$$
\lim _{t \rightarrow \infty}(\tilde{v}, \tilde{w})=(0,0)
$$


where

$$
\tilde{v}:=v-v^{*}, \quad \tilde{\omega}:=\omega-\omega^{*} .
$$

Depending on the conditions on the reference trajectories $v_{r}$ and $\omega_{r}$ we identify the following mutually-exclusive scenarios:

Tracking scenario (S1): it is assumed that there exist $T$ and $\mu>0$ such that

$$
\int_{t}^{t+T}\left[\left|v_{r}(\tau)\right|^{2}+\left|\omega_{r}(\tau)\right|^{2}\right] d \tau \geq \mu \quad \forall t \geq 0 .
$$

Stabilization scenario (S2): it is assumed that $\left|v_{r}(t)\right|+\left|\omega_{r}(t)\right| \rightarrow 0$ and there exists $\beta>0$ such that, for all $t \geq t_{\circ}$ :

$$
\int_{t_{\circ}}^{t}\left[\left|v_{r}(\tau)\right|+\left|\omega_{r}(\tau)\right|\right] d \tau<\beta, \quad \forall t \geq t_{\circ} .
$$

\subsection{Main result}

Under the conditions described above, we design a controller that achieves the trajectory tracking objective (6), (7) in either of the two mutually-exclusive scenarios described above. Our contributions are the following:

- we propose a class of control inputs $v^{*}$ and $\omega^{*}$ that extends the controller proposed in Wang et al. (2015) and we ensure uniform global asymptotic stability of the origin for (5);

- for the velocity error kinematics in closed loop, we establish integral input-tostate stability with respect to the error velocities $[\tilde{v}, \tilde{\omega}]$;

- for any control inputs $u_{1}$ and $u_{2}$ ensuring that $\tilde{v} \rightarrow 0$ and $\tilde{\omega} \rightarrow 0$, we establish global attractivity of the origin provided that the error velocities converge sufficiently fast (they are square-integrable).

The control laws that ensure the properties above are:

$$
\begin{aligned}
v^{*} & :=v_{r}(t) \cos \left(e_{\theta}\right)+k_{x} e_{x} \\
\omega^{*} & :=\omega_{r}+k_{\theta} e_{\theta}+k_{y} e_{y} v_{r} \phi\left(e_{\theta}\right)+\rho(t) k_{y} f\left(t, e_{x}, e_{y}\right)
\end{aligned}
$$

where $\phi$ is the so-called 'sinc' function defined by

$$
\begin{gathered}
\phi\left(e_{\theta}\right):=\frac{\sin \left(e_{\theta}\right)}{e_{\theta}} \\
\rho(t):=\exp \left(-\int_{0}^{t}\left[\left|v_{r}(\tau)\right|+\left|\omega_{r}(\tau)\right|\right] d \tau\right),
\end{gathered}
$$

and $f: \mathbb{R}_{\geq 0} \times \mathbb{R}^{2} \rightarrow \mathbb{R}$ is a continuously differentiable function defined such that the following hypotheses hold. 
A1. There exist a non-decreasing function $\sigma_{1}: \mathbb{R}_{\geq 0} \rightarrow \mathbb{R}_{\geq 0}$ and $\sigma_{2}>0$ such that

$$
\begin{aligned}
\max \left\{\frac{\partial f}{\partial t}, \frac{\partial f}{\partial e_{x}}, \frac{\partial f}{\partial e_{y}}\right\} & \leq \sigma_{1}\left(\left|\left[e_{x} e_{y}\right]\right|\right) \\
\left|f\left(t, e_{x}, e_{y}\right)\right| & \leq \sigma_{2}\left|\left[e_{x} e_{y}\right]\right|
\end{aligned}
$$

A2. For the function

$$
f_{\circ}\left(t, e_{y}\right):=f\left(t, 0, e_{y}\right)
$$

we assume that $\partial f_{\circ} / \partial t$ is uniform $\delta$-persistently exciting with respect to $e_{y}$ that is, for any $\delta>0$ there exist $\mu_{\delta}$ and $T_{\delta}>0$ such that

$$
\left|e_{y}\right| \geq \delta \Longrightarrow \int_{t}^{t+T_{\delta}}\left|\frac{\partial f_{\circ}}{\partial t}\left(\tau, e_{y}\right)\right| d \tau \geq \mu_{\delta} \quad \forall t \geq 0
$$

- cf. (Loría, Panteley, Popovic, \& Teel, 2002, Definition 3). Roughly speaking, the purpose of the function $f$ is to excite the $e_{y}$-dynamics as long as $\left|e_{y}\right|$ is separated from zero.

The controller (12), which achieves both the tracking and the stabilization control goals, is a weighted sum of the tracking controller

$$
\omega_{t r a}^{*}:=\omega_{r}+k_{\theta} e_{\theta}+k_{y} e_{y} v_{r} \phi\left(e_{\theta}\right),
$$

and the stabilization controller

$$
\omega_{s t a b}^{*}:=\omega_{r}+k_{\theta} e_{\theta}+k_{y} f\left(t, e_{x}, e_{y}\right)
$$

—cf. Miao and Wang (2015); Wang et al. (2015). The weight function $\rho(t)$ promotes the application of either $\omega_{t r a}^{*}$ or $\omega_{s t a b}^{*}$, depending on the task scenario S1 or S2. More precisely, from (13) we see that $\rho$ satisfies

$$
\dot{\rho}=-\left[\left|v_{r}(t)\right|+\left|\omega_{r}(t)\right|\right] \rho
$$

and $\rho \rightarrow 0$ exponentially fast if (9) holds. Hence, the tracking scenario $\mathbf{S} \mathbf{1}$ is favoured. If, instead, (10) holds, the reference velocities converge and $\rho(t)>\exp (-\beta)$. Hence, the action of the stabilization controller is enhanced.

Remark 1. The idea of so merging the two controllers for the two scenarios S1 and S2 was introduced in Wang et al. (2015). The class of controllers satisfying A1-A2 covers those in Wang et al. (2015); in particular, the function $f$ is not necessarily globally bounded and may depend only on $e_{y}$. A more significant contribution with respect to the literature is that we establish uniform global asymptotic stability for (5) in closed-loop with $(v, \omega)=\left(v^{*}, \omega^{*}\right)$; this is in contrast with Wang et al. (2015) and Do, Jiang, and Pan (2004a) where it is proved that the convergence property (6) holds. In addition, we establish integral-input-to-state stability of (5) with respect to $[\tilde{v}, \tilde{\omega}]$.

Proposition 1 (Main result). Consider the system (5) with $v=\tilde{v}+v^{*}, \omega=\tilde{\omega}+\omega^{*}$, and the virtual inputs (11) and (12). Let $k_{x}, k_{\theta}$, and $k_{y}>0$. Assume that there exist 
$\bar{\omega}_{r}, \overline{\dot{\omega}}_{r}, \bar{v}_{r}, \overline{\dot{v}}_{r}>0$ such that ${ }^{2}$

$$
\left|\omega_{r}\right|_{\infty} \leq \bar{\omega}_{r}, \quad\left|\dot{\omega}_{r}\right|_{\infty} \leq \overline{\dot{\omega}}_{r}, \quad\left|v_{r}\right|_{\infty} \leq \bar{v}_{r}, \quad\left|\dot{v}_{r}\right|_{\infty} \leq \overline{\dot{v}}_{r}
$$

Furthermore, assume that $\boldsymbol{A} \mathbf{1}$ - $\boldsymbol{A} 2$ hold.

Then, if either (9) or (10) hold the closed-loop system resulting from (5), (8), (11), and (12) has the following properties:

(P1) if $\tilde{v}=\tilde{\omega}=0$, the origin $\{e=0\}$ is uniformly globally asymptotically stable;

(P2) the closed-loop system is integral input-to-state stable with respect to $\eta:=[\tilde{v} \tilde{\omega}]^{\top}$; (P3) if $\eta \rightarrow 0$ and $\eta \in \mathcal{L}_{2}$, then (6) holds.

The proof is presented in Section 3. Below, we present an example of an adaptive controller that ensures that $\tilde{v}, \tilde{\omega} \rightarrow 0$ for any once continuously differentiable $v^{*}, \omega^{*}$.

\subsection{Example}

As in Do (2007), we consider mobile robots modeled by

$$
\begin{aligned}
& \dot{z}=J(z) \nu \\
& M \dot{\nu}+C(\dot{z}) \nu=\tau
\end{aligned}
$$

where $z:=\left[\begin{array}{lll}x & y & \theta\end{array}\right]$ contains the Cartesian coordinates $(x, y)$ and the orientation $\theta$ of the robot, $\tau \in \mathbb{R}^{2}$ corresponds to the (torque) control input; $\nu:=\left[\begin{array}{ll}\nu_{1} & \nu_{2}\end{array}\right]$ stands for the angular velocities corresponding to the two robot's wheels, $M$ is the inertia matrix, which is constant, symmetric and positive definite, and $C(\dot{z})$ is the matrix of Coriolis forces, which is skew-symmetric. In addition, we use the coordinate transformation matrix

$$
J(z)=\frac{r}{2}\left[\begin{array}{cc}
\cos (\theta) & \cos (\theta) \\
\sin (\theta) & \sin (\theta) \\
1 / b & -1 / b
\end{array}\right]
$$

where $r$ is the radius of either steering wheel and $b$ is the distance from the center of either wheel to the Cartesian point $(x, y)$. The relation between the wheels' velocities, $\nu$, and the robot's velocities in the fixed frame, $\dot{z}$, is given by

$$
\left[\begin{array}{c}
v \\
\omega
\end{array}\right]:=\frac{r}{2 b}\left[\begin{array}{cc}
b & b \\
1 & -1
\end{array}\right]\left[\begin{array}{l}
\nu_{1} \\
\nu_{2}
\end{array}\right] \Leftrightarrow\left[\begin{array}{l}
\nu_{1} \\
\nu_{2}
\end{array}\right]=\frac{1}{r}\left[\begin{array}{cc}
1 & b \\
1 & -b
\end{array}\right]\left[\begin{array}{c}
v \\
\omega
\end{array}\right]
$$

which may be used in (19a) to obtain the familiar model (1).

We assume that the inertia parameters and the constants contained in $C(\dot{z})$ are unknown while $r$ and $b$ are considered to be known. Let $\hat{M}$ and $\hat{C}$ denote, respectively, the estimates of $M$ and $C$. Furthermore, let $\nu^{*}:=\left[\nu_{1}^{*} \nu_{2}^{*}\right]^{\top}$,

$$
\left[\begin{array}{c}
\nu_{1}^{*} \\
\nu_{2}^{*}
\end{array}\right]=\frac{1}{r}\left[\begin{array}{cc}
1 & b \\
1 & -b
\end{array}\right]\left[\begin{array}{c}
v^{*} \\
\omega^{*}
\end{array}\right]
$$

\footnotetext{
${ }^{2}$ For a continuous function $t \mapsto \varphi$ we define $|\varphi(t)|_{\infty}:=\sup _{t \geq 0}|\varphi(t)|$.
} 
and let us introduce the certainty-equivalence control law

$$
\tau^{*}:=\hat{M} \dot{\nu}^{*}+\hat{C}(\dot{z}) \nu^{*}-k_{d} \sigma(\tilde{\nu}), \quad k_{d}>0
$$

where $\tilde{\nu}:=\nu-\nu^{*}$ and $\sigma(\cdot)$ is a continuous locally linear odd function for which there exist $\epsilon_{1}$ and $\epsilon_{2}>0$ such that $\sigma(s)^{\top} s \geq \epsilon_{1}|s|^{2}$ for all $|s| \leq \epsilon_{2}$ as, for instance, $\sigma(s)=s$, $\sigma(s)=\left[\tanh \left(s_{1}\right) \cdots \tanh \left(s_{n}\right)\right]^{\top}$, and many other saturation functions.

Then, let us define $\tilde{M}:=\hat{M}-M$ and $\tilde{C}:=\hat{C}-C$, so

$$
\tau^{*}:=M \dot{\nu}^{*}+C(\dot{z}) \nu^{*}-k_{d} \sigma(\tilde{\nu})+\tilde{M} \dot{\nu}^{*}+\tilde{C} \nu^{*}
$$

and, setting $\tau=\tau^{*}$ in (19b), we obtain the closed-loop equation

$$
M \dot{\tilde{\nu}}+C(\dot{z}) \tilde{\nu}+k_{d} \sigma(\tilde{\nu})=\Psi\left(\dot{z}, \dot{\nu}^{*}, \nu^{*}\right)^{\top} \tilde{\Theta}
$$

where $\Theta \in \mathbb{R}^{m}$ is a vector of constant (unknown) lumped parameters in $M$ and $C$, $\hat{\Theta}$ denotes the estimate of $\Theta, \tilde{\Theta}:=\hat{\Theta}-\Theta$ is the vector of estimation errors, and $\Psi: \mathbb{R}^{3} \times \mathbb{R}^{2} \times \mathbb{R}^{2} \rightarrow \mathbb{R}^{m \times 2}$ is a continuous known function. To obtain (24), we used the property that (19b) is linear in the constant lumped parameters. In addition, we use the passivity-based adaptation law - cf. Ortega and Spong (1989),

$$
\dot{\hat{\Theta}}=-\gamma \Psi\left(\dot{z}, \dot{\nu}^{*}, \nu^{*}\right) \tilde{\nu}, \quad \gamma>0 \text {. }
$$

Then, a direct computation shows that the total derivative of

$$
V(\tilde{\nu}, \tilde{\Theta}):=\frac{1}{2}\left[|\tilde{\nu}|^{2}+\frac{1}{\gamma}|\tilde{\Theta}|^{2}\right]
$$

along the trajectories of (24), (25), yields

$$
\dot{V}(\tilde{\nu}, \tilde{\Theta}) \leq-k_{d} \sigma(\tilde{\nu})^{\top} \tilde{\nu}
$$

which implies that $\dot{V}(\tilde{\nu}(t), \tilde{\Theta}(t)) \leq 0$. Integrating the latter from 0 to infinity, we obtain that $\tilde{\Theta}, \tilde{\nu} \in \mathcal{L}_{\infty}$. Then, there exists $\epsilon_{2}>0$ such that $|\tilde{\nu}(t)| \leq \epsilon_{2}$ for all $t \geq 0$ and, therefore, there also exists $\epsilon_{1}\left(\epsilon_{2}\right)>0$ such that

$$
\left.\dot{V}(\tilde{\nu}(t), \tilde{\Theta}(t)) \leq-k_{d} \mid \tilde{\nu}(t)\right)\left.\right|^{2} .
$$

Integrating the latter from 0 to infinity we obtain that $\tilde{\nu} \in \mathcal{L}_{2}$. It follows, e.g., from (Ioannou \& Sun, 1996, Lemma 3.2.5), that $\tilde{\nu} \rightarrow 0$ and, in view of (20),

$$
\lim _{t \rightarrow \infty}|\tilde{v}(t)|+|\tilde{\omega}(t)|=0
$$

Also, in view of $(20), \tilde{\nu} \in \mathcal{L}_{2}$ implies that $\eta \in \mathcal{L}_{2}$.

\section{Proof of the main result}

For each scenario, $\mathbf{S 1}$ and $\mathbf{S 2}$ we establish uniform global asymptotic stability for the closed-loop kinematics equation (5) restricted to $\eta=0(\mathbf{P 1})$. Then, we establish the 
iISS with respect to $\eta(\mathbf{P 2})$ by showing that the closed-loop trajectories are bounded, under the condition that $\eta$ is square integrable — cf. Angeli et al. (2004).

\subsection{Under Scenario $\mathrm{S} 1$}

The proof of Proposition 1 under condition (9) is constructive; we provide a strict Lyapunov function. To that end, we start by observing that the error system (5), (8), (11) and (12) takes the form

$$
\dot{e}=A_{v_{r}}(t, e) e+B_{1}(t, e) \rho(t)+B_{2}(e) \eta
$$

where $\rho(t)$ is defined in (13),

$$
\begin{aligned}
A_{v_{r}}(t, e) & :=\left[\begin{array}{ccc}
-k_{\theta} & 0 & -v_{r}(t) k_{y} \phi\left(e_{\theta}\right) \\
0 & -k_{x} & \varphi(t, e) \\
v_{r}(t) \phi\left(e_{\theta}\right) & -\varphi(t, e) & 0
\end{array}\right], \\
B_{1}(t, e) & :=\left[\begin{array}{c}
-k_{y} f\left(t, e_{x}, e_{y}\right) \\
k_{y} f\left(t, e_{x}, e_{y}\right) e_{y} \\
-k_{y} f\left(t, e_{x}, e_{y}\right) e_{x}
\end{array}\right],
\end{aligned}
$$

where $\varphi(t, e):=\omega_{r}(t)+k_{\theta} e_{\theta}+k_{y} e_{y} v_{r}(t) \phi\left(e_{\theta}\right)$. Writing the closed-loop dynamics as in (27) is convenient to stress that the "nominal" system $\dot{e}=A_{v_{r}}(t, e) e$ has a familiar structure encountered in model reference adaptive control. Moreover, defining

$$
V_{1}(e):=\frac{1}{2}\left[e_{x}^{2}+e_{y}^{2}+\frac{1}{k_{y}} e_{\theta}^{2}\right]
$$

we obtain, along the trajectories of $\dot{e}=A_{v_{r}}(t, e) e$,

$$
\dot{V}_{1}(e) \leq-k_{x} e_{x}^{2}-\frac{k_{\theta}}{k_{y}} e_{\theta}^{2}
$$

This is a fundamental first step for the design of a strict Lyapunov function for the "perturbed" system (27), using the Mazenc construction method.

Now, to establish the proof in the case of scenario $\mathbf{S 1}$, we follow the following steps:

Step 1) We build a strict Lyapunov function $V(t, e)$ for the nominal system $\dot{e}=$ $A_{v_{r}}(t, e) e$. This establishes $\mathbf{P} \mathbf{1}$.

Step 2) We construct a function $W(t, e)$ for the perturbed system $\dot{e}=A_{v_{r}}(t, e) e+$ $B_{1}(t, e) \rho$.

Step 3) We use $W(t, e)$ to prove integral ISS of (27) with respect to $\eta$ (i.e., P2) as well as the boundedness of the trajectories under the assumption that $\eta \in \mathcal{L}_{2}$. This and the assumption that $\eta \rightarrow 0$ implies (6), i.e., P3.

Step 1. We establish UGAS for the nominal system

$$
\dot{e}=A_{v_{r}}(t, e) e
$$

via Lyapunov's direct method ${ }^{3}$. Let $F_{[3]}, S_{[3]}: \mathbb{R}_{\geq 0} \rightarrow \mathbb{R}_{\geq 0}$, and $P_{[k]}: \mathbb{R}_{\geq 0} \times \mathbb{R}_{\geq 0} \rightarrow \mathbb{R}_{\geq 0}$

${ }^{3}$ This proof of uniform stability replaces the corresponding one proposed in Maghenem, Loría, and Panteley (2017), which is incorrect. 
be smooth polynomials in $V_{1}$ with strictly positive and bounded coefficients of degree 3 and $k$ respectively. After (Maghenem, Loría, \& Panteley, 2016, Proposition 1), there exists a positive definite radially unbounded function $V: \mathbb{R}_{\geq 0} \times \mathbb{R}^{3} \rightarrow \mathbb{R}_{\geq 0}$ defined as

$$
\begin{aligned}
V(t, e):=\quad P_{[2]}\left(t, V_{1}\right) V_{1}(e)-\omega_{r}(t) e_{x} e_{y} \\
+v_{r}(t) P_{[1]}\left(t, V_{1}\right) e_{\theta} e_{y}
\end{aligned}
$$

and such that

$$
F_{[3]}\left(V_{1}\right) \leq V(t, e) \leq S_{[3]}\left(V_{1}\right),
$$

where $V_{1}$ is defined in (29), It is showed in Maghenem et al. (2016) that the total derivative of $V$ along the trajectories of (30) satisfies

$$
\dot{V}(t, e) \leq-\frac{\mu}{T} V_{1}(e)-k_{x} e_{x}^{2}-\frac{k_{\theta}}{k_{y}} e_{\theta}^{2}
$$

Hence uniform global asymptotic stability of the null solution of (30) follows.

Step 2. Now we construct a strict Lyapunov function for the system

$$
\dot{e}=A_{v_{r}}(t, e) e+B_{1}(t, e) \rho(t) .
$$

To that end, we start by "reshaping" the function $V$ defined in (31) to obtain a particular negative bound on its time derivative. Let

$$
Z(t, e):=Q_{[3]}\left(V_{1}\right) V_{1}(e)+V(t, e)
$$

where $Q_{[3]}\left(V_{1}\right)$ is a third order polynomial of $V_{1}$, with strictly positive coefficients. Then, in view of (33), the total derivative of $Z$ along the trajectories of (30) satisfies

$$
\dot{Z}(t, e) \leq-\frac{\mu}{T} V_{1}(e)-Q_{[3]}\left(V_{1}\right)\left[k_{x} e_{x}^{2}+\frac{k_{\theta}}{k_{y}} e_{\theta}^{2}\right] .
$$

Next, we recall that in view of $(9) \rho(t)$, which satisfies (18) is uniformly integrable. Therefore, for any $\gamma>0$, there exists $c>0$ such that

$$
G(t):=\exp \left(-\gamma \int_{0}^{t} \rho(s) d s\right) \geq c>0 \quad \forall t \geq 0
$$

and, since $Z(t, e)$ and $V(t, e)$ are positive definite radially unbounded - see (32) and (35), so is the function

$$
W(t, e):=G(t) Z(t, e)
$$

indeed, we have

$$
\exp \left(-\gamma \int_{0}^{\infty} \rho(s) d s\right) Z(t, e) \leq W(t, e) \leq Z(t, e)
$$


Now, the time derivative of $W$ along trajectories of (34) verifies

$$
\begin{aligned}
\dot{W}(t, e) \leq & -Y(t, e)+\dot{G}(t) Z(t, e) \\
& +G(t) \frac{\partial\left(V+Q_{[3]}\left(V_{1}\right) V_{1}\right)}{\partial e} B_{1}(t, e) \rho(t) \\
Y(t, e):= & G(t)\left[\frac{\mu}{T} V_{1}(e)+Q_{[3]}\left(V_{1}\right)\left[k_{x} e_{x}^{2}+\frac{k_{\theta}}{k_{y}} e_{\theta}^{2}\right]\right] .
\end{aligned}
$$

Note that, in view of $(37), Y(t, e)$ is positive definite. We proceed to show that the rest of the terms bounding $\dot{W}$ are negative semi-definite. To that end, we develop (dropping the arguments of $f\left(t, e_{x}, e_{y}\right)$ )

$$
\begin{aligned}
\frac{\partial V}{\partial e} B_{1}(t, e)= & \frac{\partial V}{\partial V_{1}} \frac{\partial V_{1}}{\partial e} B_{1}(t, e)-\omega_{r} k_{y} f(\cdot)\left[e_{x}+e_{y}^{2}\right] \\
& -v_{r} P_{[1]}\left(t, V_{1}\right) k_{y} f(\cdot)\left[e_{\theta} e_{x}+e_{y}\right]
\end{aligned}
$$

and

$$
\frac{\partial\left(Q_{[3]}\left(V_{1}\right) V_{1}\right)}{\partial e} B_{1}(t, e)=\frac{\partial\left(Q_{[3]}\left(V_{1}\right) V_{1}\right)}{\partial V_{1}} \frac{\partial V_{1}}{\partial e} B_{1}(t, e),
$$

and we decompose $B_{1}(t, e)$ into

$$
B_{1}(t, e)=\left[\begin{array}{c}
-k_{y} f(\cdot) \\
0 \\
0
\end{array}\right]+\left[\begin{array}{ccc}
0 & 0 & 0 \\
0 & 0 & k_{y} f(\cdot) \\
0 & -k_{y} f(\cdot) & 0
\end{array}\right] e .
$$

Then, since

$$
\frac{\partial V_{1}}{\partial e}\left[\begin{array}{ccc}
0 & 0 & 0 \\
0 & 0 & k_{y} f(\cdot) \\
0 & -k_{y} f(\cdot) & 0
\end{array}\right] e=0
$$

it follows that

$$
\frac{\partial V_{1}}{\partial e} B_{1}(t, e)=-\frac{\partial V_{1}}{\partial e_{\theta}} k_{y} f(\cdot)=-e_{\theta} f(\cdot)
$$

Thus, using the latter equation, we obtain

$$
\begin{aligned}
& \dot{W}(t, e) \leq-Y(t, e)+\dot{G}(t) Z(t, e) \\
& \quad-G(t) \rho(t) f(\cdot) \frac{\partial\left(V+Q_{[3]}\left(V_{1}\right) V_{1}\right)}{\partial V_{1}} e_{\theta} \\
& \quad+v_{r} f(\cdot) G(t) \rho(t) P_{[1]}\left(t, V_{1}\right)\left[-k_{y} e_{\theta} e_{x}-k_{y} e_{y}\right] \\
& \quad+\omega_{r} G(t) \rho(t) f(\cdot)\left[-k_{y} e_{x}+k_{y} e_{y}^{2}\right] .
\end{aligned}
$$

In view of $(15)$ and the boundedness of $v_{r}$ and $\omega_{r}$, there exists a polynomial $R_{[3]}\left(V_{1}\right)$ 
with non-negative coefficients, such that

$$
\begin{aligned}
R_{[3]}\left(V_{1}\right) & V_{1} \geq-f(\cdot) \frac{\partial\left(V+Q_{[3]}\left(V_{1}\right) V_{1}\right)}{\partial V_{1}} e_{\theta} \\
& +\omega_{r} f(\cdot)\left[-k_{y} e_{x}+k_{y} e_{y}^{2}\right] \\
& +v_{r} f(\cdot) P_{[1]}\left(t, V_{1}\right)\left[-k_{y} e_{\theta} e_{x}-k_{y} e_{y}\right] .
\end{aligned}
$$

Hence, since $V(t, e) \geq F_{[3]}\left(V_{1}\right)$ — see $(32)$, we obtain

$$
\dot{W} \leq-Y(t, e)+\dot{G}(t) F_{[3]}\left(V_{1}\right) V_{1}+G(t) \rho(t) R_{[3]}\left(V_{1}\right) V_{1} .
$$

On the other hand, in view of $(37), \dot{G}(t) \leq-\gamma G(t) \rho(t)$ for any $\gamma>0$ and the coefficients of $F_{[3]}\left(V_{1}\right)$ are strictly positive. Therefore, there exists $\gamma>0$ such that

$$
\gamma F_{[3]}\left(V_{1}\right) \geq R_{[3]}\left(V_{1}\right)
$$

and, consequently, $\dot{W}(t, e) \leq-Y(t, e)$ for all $t \geq 0$ and all $e \in \mathbb{R}^{3}$. Uniform global asymptotic stability of the null solution of (34) follows.

Step 3. In order to establish iISS with respect to $\eta$ and boundedness of the closedloop trajectories subject to $\eta \in \mathcal{L}_{2}$, we proceed as in (Maghenem et al., 2016, Proposition 4). Let

$$
W_{1}(t, e):=\ln (1+W(t, e)) .
$$

The derivative of $W_{1}$ along trajectories of (27) satisfies

$$
\dot{W}_{1} \leq-G_{m} \frac{\frac{\mu}{T} V_{1}(e)+Q_{[3]}\left[k_{x} e_{x}^{2}+\frac{k_{\theta}}{k_{y}} e_{\theta}^{2}\right]}{1+W(t, e)}+\frac{\left|\frac{\partial W}{\partial e} B_{2} \eta\right|}{1+W(t, e)}
$$

with $G_{m}:=\exp \left(-\gamma \int_{0}^{\infty} \rho(t) d t\right)$.

Next, we decompose $B_{2}(e) \eta$ introduced in (27) into

$$
B_{2}(e) \eta:=B_{21}(\eta)+B_{22}(\eta) e
$$

where

$$
B_{21}(\eta):=\left[\begin{array}{c}
-\tilde{\omega} \\
-\tilde{v} \\
0
\end{array}\right], \quad B_{22}(\eta):=\left[\begin{array}{ccc}
0 & 0 & 0 \\
0 & 0 & \tilde{\omega} \\
0 & -\tilde{\omega} & 0
\end{array}\right]
$$

Then, we use the fact that $\frac{\partial V_{1}}{\partial e} B_{22}(\eta) e=0$ and $|G(t)| \leq 1$, and we define

$$
H\left(e, V_{1}\right):=Q_{[3]}+P_{[3]}+\frac{\partial Q_{[3]}}{\partial V_{1}} V_{1}+\frac{\partial P_{[3]}}{\partial V_{1}} V_{1}+\bar{v}_{r}\left|e_{\theta}\right|\left|e_{y}\right| \frac{\partial P_{[1]}}{\partial V_{1}}
$$


and

$$
\xi=\left[\begin{array}{l}
\frac{e_{\theta}}{k_{y}} \\
e_{x}
\end{array}\right],
$$

to obtain

$$
\begin{aligned}
\left|\frac{\partial W}{\partial e} B_{2} \eta\right| \leq & H\left(e, V_{1}\right)|\xi||\eta|+\bar{\omega}_{r}\left|e_{y}\right||\eta|+\bar{v}_{r} P_{[1]}\left|e_{y}\right||\eta| \\
& +\bar{\omega}_{r} V_{1}|\eta|+\bar{v}_{r} P_{[1]}\left|e_{\theta}\right|\left|e_{x}\right||\eta| \\
\leq & H\left(e, V_{1}\right)\left[\frac{1}{2 \epsilon}|\xi|^{2}+\frac{\epsilon}{2}|\eta|^{2}\right]+\bar{\omega}_{r}\left[\frac{1}{2 \epsilon} V_{1}+\frac{\epsilon}{2} V_{1}|\eta|^{2}\right] \\
& +\bar{\omega}_{r}\left[\frac{1}{2 \epsilon} V_{1}+\frac{\epsilon}{2}|\eta|^{2}\right]+\bar{v}_{r}\left[\frac{1}{2 \epsilon} V_{1}+\frac{\epsilon}{2} P_{[1]}^{2}|\eta|^{2}\right] \\
& \quad \bar{v}_{r} P_{[1]}\left[\frac{1}{2 \epsilon} V_{1}\left|e_{\theta}\right|^{2}+\frac{\epsilon}{2}|\eta|^{2}\right] \\
\leq & \left.H\left(e, V_{1}\right)+\bar{v}_{r} P_{[1]} k_{y}^{2} V_{1}\right] \frac{1}{2 \epsilon}|\xi|^{2}+\left[2 \bar{\omega}_{r}+\bar{v}_{r}\right] \frac{1}{2 \epsilon} V_{1} \\
+ & \frac{\epsilon}{2}|\eta|^{2}\left[H\left(e, V_{1}\right)+\bar{\omega}_{r} V_{1}+\bar{\omega}_{r}+\bar{v}_{r} P_{[1]}^{2}+\bar{v}_{r} P_{[1]}\right] .
\end{aligned}
$$

Next, we choose $\epsilon>0$ such that

$$
\begin{aligned}
\frac{H+\bar{v}_{r} P_{[1]} k_{y}^{2} V_{1}}{\epsilon}|\xi|^{2} & \leq G_{m} Q_{[3]}\left[k_{x} e_{x}^{2}+\frac{k_{\theta}}{k_{y}} e_{\theta}^{2}\right], \\
\frac{2 \bar{\omega}_{r}+\bar{v}_{r}}{\epsilon} & \leq G_{m} \frac{\mu}{T} .
\end{aligned}
$$

Such $\epsilon>0$ exists because $Q_{[3]}$ is a third order polynomial of $V_{1}$ with strictly positive coefficients. So (46) becomes

$$
\begin{aligned}
\dot{W}_{1} \leq & -\frac{G_{m}}{2} \frac{\frac{\mu}{T} V_{1}(e)+Q_{[3]}\left[k_{x} e_{x}^{2}+\frac{k_{\theta}}{k_{y}} e_{\theta}^{2}\right]}{1+W(t, e)} \\
& +\frac{D_{[3]}\left(V_{1}\right)}{1+W(t, e)} \frac{\epsilon}{2}|\eta|^{2}
\end{aligned}
$$

where $D_{[3]}\left(V_{1}\right)$ is a third order polynomial satisfying

$$
H+\bar{\omega}_{r} V_{1}+\bar{\omega}_{r}+\bar{v}_{r} P_{[1]}^{2}+\bar{v}_{r} P_{[1]} \leq D_{[3]} .
$$

From the positivity of $V,(32)$, and the definition of $W$ in (38), we have

$$
G_{m} Q_{[3]}\left(V_{1}\right) V_{1} \leq W(t, e) \leq S_{[3]}\left(V_{1}\right) V_{1}
$$


hence,

$$
\begin{aligned}
\dot{W}_{1} \leq- & \frac{G_{m}}{2} \frac{\frac{\mu}{T} V_{1}+Q_{[3]}\left(V_{1}\right)\left[k_{x} e_{x}^{2}+\frac{k_{\theta}}{k_{y}} e_{\theta}^{2}\right]}{1+S_{[3]}\left(V_{1}\right)} \\
& +\frac{D_{[3]}\left(V_{1}\right)}{1+G_{m} Q_{[3]}\left(V_{1}\right) V_{1}} \frac{\epsilon}{2}|\eta|^{2} .
\end{aligned}
$$

This implies the existence of a positive constant $c>0$ and a positive definite function $e \mapsto \alpha$ such that

$$
\dot{W}_{1} \leq-\alpha(e)+c|\eta|^{2} .
$$

The result follows from Angeli et al. (2000).

\subsection{Under the scenario S2:}

The proof of Proposition 1 under condition (10) relies on arguments for stability of cascaded systems as well as on tools tailored for systems with persistency of excitation. We start by rewriting the closed-loop equations in a convenient form for the analysis under the conditions of Scenario 2. To that end, to compact the notation, let us introduce

$$
\begin{aligned}
f_{\rho}\left(t, e_{x}, e_{y}\right) & :=\rho(t) f\left(t, e_{x}, e_{y}\right) \\
\Phi\left(t, e_{\theta}, e_{x}, e_{y}\right) & =k_{\theta} e_{\theta}+k_{y} f_{\rho}\left(t, e_{x}, e_{y}\right)
\end{aligned}
$$

Then, the closed-loop equations become

$$
\dot{e}=f_{e}(t, e)+g(t, e) \eta, \quad \eta=\left[\begin{array}{ll}
\tilde{v} & \tilde{\omega}
\end{array}\right]^{\top},
$$

where

$$
\begin{aligned}
f_{e}(t, e):= & {\left[\begin{array}{c}
-k_{\theta} e_{\theta}-k_{y} f_{\rho}-k_{y} v_{r} \phi\left(e_{\theta}\right) e_{y} \\
-k_{x} e_{x}+\Phi e_{y}+\left[\omega_{r}+k_{y} v_{r} \phi\left(e_{\theta}\right) e_{y}\right] e_{y} \\
-\Phi e_{x}-\left[\omega_{r}+k_{y} v_{r} \phi\left(e_{\theta}\right) e_{y}\right] e_{x}+v_{r} \sin e_{\theta}
\end{array}\right], } \\
g(t, e):= & {\left[\begin{array}{cc}
0 & -1 \\
-1 & e_{y} \\
0 & -e_{x}
\end{array}\right] . }
\end{aligned}
$$

Following the proof-lines of (Panteley \& Loría, 2001, Lemma 1) for cascaded systems, we establish the following for the system (54):

Claim 1. The solutions are uniformly globally bounded subject to $\eta \in \mathcal{L}_{2}$,

Claim 2. The origin of $\dot{e}=f_{e}(t, e)$ is uniformly globally asymptotically stable (i.e., P1).

After Angeli et al. (2004) the last two claims together imply integral ISS with respect to $\eta$ (i.e., P2). Moreover, Claim 1 implies the convergence of the closedloop trajectories to the origin provided that the input $\eta$ tends to zero and is square integrable (i.e., P3). 


\subsubsection{Proof of Claim 1}

Let

$$
W(e):=\ln \left(1+V_{1}(e)\right), \quad V_{1}(e):=\frac{1}{2}\left[e_{x}^{2}+e_{y}^{2}\right] .
$$

The total derivative of $V_{1}$ above along the trajectories of (54) yields

$$
\dot{V}_{1}(e) \leq-k_{x} e_{x}^{2}+\left|e_{x}\right||\tilde{v}|+\left|v_{r}\right|\left|\sin \left(e_{\theta}\right)\right|\left|e_{y}\right|
$$

hence,

$$
\begin{aligned}
\dot{W}(e) & \leq \frac{1}{1+V_{1}}\left[-\frac{k_{x}}{2} e_{x}^{2}+\left|v_{r}\right|\left|e_{y}\right|+\frac{\tilde{v}^{2}}{2 k_{x}}\right] \\
& \leq \frac{\left|e_{y}\right|}{1+V_{1}}\left|v_{r}\right|+\frac{1}{2 k_{x}\left[1+V_{1}\right]} \tilde{v}^{2} .
\end{aligned}
$$

Integrating on both sides of (58) along the trajectories, from 0 to $t$, and invoking the integrability of $v_{r}$ and the square integrability of $\eta$ we see that $W(e(t))$ is bounded for all $t \geq 0$. Boundedness of $e_{x}(t)$ and $e_{y}(t)$ follows since $W$ is positive definite and radially unbounded in $\left(e_{x}, e_{y}\right)$.

Next, we observe that the $\dot{e}_{\theta}$-equation in (54) corresponds to an exponentially stable system with bounded input $u(t)=-k_{y} v_{r}(t) \phi\left(e_{\theta}(t)\right) e_{y}(t)-k_{y} f_{\rho}\left(t, e_{x}(t), e_{y}(t)\right)-\tilde{\omega}(t)$ hence, we also have $e_{\theta} \in \mathcal{L}_{\infty}$.

Remark 2. For further development, we also emphasize that proceeding as above from Inequality (57) we conclude that $e_{x} \in \mathcal{L}_{2}$, uniformly in the initial conditions.

\subsubsection{Proof of Claim 2}

We split the drift of the nominal system $\dot{e}=f_{e}(t, e)$ into the output injection form:

$$
f_{e}(t, e)=F(t, e)+K(t, e)
$$

where

$$
F(t, e):=\left[\begin{array}{c}
-k_{\theta} e_{\theta}-k_{y} f_{\rho}\left(t, e_{x}, e_{y}\right) \\
-k_{x} e_{x}+\Phi\left(t, e_{\theta}, e_{x}, e_{y}\right) e_{y} \\
-\Phi\left(t, e_{\theta}, e_{x}, e_{y}\right) e_{x}
\end{array}\right]
$$

and

$$
K(t, e):=\left[\begin{array}{c}
-k_{y} v_{r} \phi\left(e_{\theta}\right) e_{y} \\
{\left[\omega_{r}+k_{y} v_{r} \phi\left(e_{\theta}\right) e_{y}\right] e_{y}} \\
-\left[\omega_{r}+k_{y} v_{r} \phi\left(e_{\theta}\right) e_{y}\right] e_{x}+v_{r} \sin e_{\theta}
\end{array}\right] .
$$

Then, to establish UGAS for the origin of $\dot{e}=f_{e}(t, e)$ we invoke the output-injection statement (Panteley, Loría, \& Teel, 2001, Proposition 3). According to the latter, UGAS follows if:

a) there exist: an "output" $y$, non decreasing functions $k_{1}, k_{2}$, and $\beta: \mathbb{R}_{\geq 0} \rightarrow \mathbb{R}_{\geq 0}$, a class $\mathcal{K}_{\infty}$ function $k$, and a positive definite function $\gamma$ such that, for all $t \geq 0$ and all 
$e \in \mathbb{R}^{3}$,

$$
\begin{gathered}
|K(t, e)| \leq k_{1}(|e|) k(|y|) \\
|y(t, e)| \leq k_{2}(|e|) \\
\int_{0}^{\infty} \gamma(|y(t)|) d t \leq \beta(|e(0)|) ;
\end{gathered}
$$

b) the origin of $\dot{e}=f_{e}(t, e)$ is uniformly globally stable;

c) the origin of $\dot{e}=F(t, e)$ is UGAS.

Condition a. Using (60), a direct computation shows that there exists $c>0$ such that

$$
|K(t, e)| \leq c\left[|e|^{2}+|e|\right]\left|\left[v_{r} \omega_{r}\right]\right|,
$$

so (61) holds with $k_{1}(s):=c\left(s^{2}+s\right), k(s):=s$, and $y:=\left[v_{r} \omega_{r}\right]$. Moreover, (62) and (63) hold with $\gamma(s)=s$, since $\left[v_{r} \omega_{r}\right] \in \mathcal{L}_{1}$, for a constant functions $\beta$ and $k_{2}$ which, moreover, are independent of the initial state.

Condition b. Uniform global stability is tantamount to uniform stability and uniform global boundedness of the solutions — see Hahn (1967). The latter was established already for the closed-loop system under the action of the "perturbation" $\eta$ hence, it holds all the more in this case, where $\eta=0$.

In order to establish uniform stability, we use Lyapunov's direct method. Let $R>0$ be arbitrary but fixed.

We claim that, for the system $\dot{e}=F(t, e)$, there exists a Lyapunov function candidate $V: \mathbb{R}_{\geq 0} \times \mathbb{R}^{3} \rightarrow \mathbb{R}_{\geq 0}$ and positive constants $\alpha_{1}, \alpha_{2}$, and $\alpha_{3}$ such that

$$
\begin{aligned}
\alpha_{1}|e|^{2} \leq V(t, e) \leq \alpha_{2}|e|^{2} & \forall t \geq 0, e \in \mathbb{R}^{3} \\
\left|\frac{\partial V(t, e)}{\partial e}\right| \leq \alpha_{3}|e| & \forall t \geq 0, e \in \mathbb{R}^{3} \\
\frac{\partial V}{\partial t}+\frac{\partial V}{\partial e} F(t, e) \leq 0 & \forall t \geq 0, e \in B_{R}
\end{aligned}
$$

where $B_{R}:=\left\{e \in \mathbb{R}^{3}:|e| \leq R\right\}$. Furthermore, from (64) it follows that

$$
|K(t, e)| \leq c(R+1)\left[\left|v_{r}\right|+\left|\omega_{r}\right|\right]|e| \quad \forall t \geq 0, e \in B_{R} .
$$

Then, evaluating the time derivative of $V$ along the trajectories of (59), we obtain

$$
\begin{aligned}
\dot{V}(t, e) & \leq \frac{\partial V(t, e)}{\partial e} K(t, e) \leq \alpha_{3} c(R+1)\left[\left|v_{r}\right|+\left|\omega_{r}\right|\right]|e|^{2} \\
& \leq \frac{\alpha_{3} c[R+1]}{\alpha_{1}}\left[\left|v_{r}\right|+\left|\omega_{r}\right|\right] V(t, e) \quad \forall t \geq 0, e \in B_{R} .
\end{aligned}
$$

Defining $v(t):=V(t, e(t))$ and invoking the comparison lemma, we conclude that

$$
v(t) \leq \exp \left(\frac{c \alpha_{3}[R+1]}{\alpha_{1}} \int_{t_{\circ}}^{\infty}\left[\left|v_{r}(s)\right|+\left|\omega_{r}(s)\right|\right] d s\right) v\left(t_{\circ}\right)
$$

for all initial conditions $t_{\circ} \geq 0$ and $e\left(t_{\circ}\right)$ generating trajectories $e(t) \in B_{R}$. In view of 
(10), we obtain

$$
|e(t)|^{2} \leq \frac{\alpha_{2}}{\alpha_{1}} \exp \left(\frac{\alpha_{3} c[R+1]}{\alpha_{1}} \beta\right)\left|e\left(t_{\circ}\right)\right|^{2}
$$

so uniform stability of (59) follows.

It is left to construct a Lyapunov function candidate $V$ for the system $\dot{e}=F(t, e)$, that satisfies the conditions (65)-(67). To that end, consider the coordinates

$$
e_{z}=e_{\theta}+g\left(t, e_{y}\right)
$$

where $g: \mathbb{R}_{\geq 0} \times \mathbb{R}_{\geq 0} \rightarrow \mathbb{R}_{\geq 0}$ defined by

$$
g\left(t, e_{y}\right):=e^{-k_{\theta}\left(t-t_{\circ}\right)} g\left(t_{\circ}, e_{y}\right)+\int_{t_{\circ}}^{t} k_{y} e^{-k_{\theta}(t-s)} f\left(s, 0, e_{y}\right) d s
$$

and, for further development we observe that

$$
\frac{\partial g}{\partial t}\left(t, e_{y}\right)=-k_{\theta} g\left(t, e_{y}\right)+k_{y} f_{\rho}\left(t, 0, e_{y}\right) .
$$

Let $g\left(t_{\circ}, e_{y}\right)$ be such that $\left|g\left(t_{\circ}, e_{y}\right)\right| \leq\left|e_{y}\right|$ which implies, using Assumption A1, that

$$
\left|g\left(t, e_{y}\right)\right| \leq\left(1+k_{y} \sigma_{2}\right)\left|e_{y}\right|
$$

In the new coordinates, we obtain

$$
\dot{e}_{z}=-k_{\theta} e_{z}-\frac{\partial g}{\partial e_{y}} \Phi e_{x}-k_{y} \tilde{f}\left(t, e_{x}, e_{y}\right)
$$

where $\tilde{f}\left(t, e_{x}, e_{y}\right):=f_{\rho}\left(t, e_{x}, e_{y}\right)-\tilde{f}_{\rho}\left(t, 0, e_{y}\right)$. Then, Assumption A1 implies that for any $R>0$ there exists a positive constant $c_{R}>0$ such that

$$
\max _{e \in B_{R}}\left\{\sup _{t \geq 0}\left|\tilde{f}_{\rho}\left(t, e_{x}, e_{y}\right)\right|, \quad \sup _{t \geq 0}\left|\frac{\partial g}{\partial e_{y}} \Phi e_{x}\right|\right\} \leq c_{R}\left|e_{x}\right| .
$$

Thus, consider the following Lyapunov function candidate

$$
V(t, e):=\left[\frac{1}{2} \frac{c_{R}^{2}}{k_{\theta} k_{x}}+\left(1+k_{y} \sigma_{2}\right)^{2}\right]\left[e_{x}^{2}+e_{y}^{2}\right]+\frac{1}{2} e_{z}^{2}
$$

which trivially satisfies (66). Its total time derivative is

$$
\begin{aligned}
\dot{V}(t, e) & =-\frac{c_{R}^{2}}{k_{\theta}} e_{x}^{2}-e_{z}\left[k_{\theta} e_{z}+\frac{\partial g}{\partial e_{y}} \Phi e_{x}+k_{y} \tilde{f}\left(t, e_{x}, e_{y}\right)\right] \\
& \leq-\frac{c_{R}^{2}}{k_{\theta}} e_{x}^{2}-k_{\theta} e_{z}^{2}-c_{R}\left|e_{z}\right|\left|e_{x}\right| \leq 0, \quad \forall e \in B_{R},
\end{aligned}
$$


so (67) holds. Using (70) and the inequalities

$$
\begin{aligned}
& e_{z}^{2} \geq e_{\theta}^{2}-2\left|e_{\theta}\right|\left|g\left(t, e_{y}\right)\right|+\left|g\left(t, e_{y}\right)\right|^{2} \geq \frac{1}{2} e_{\theta}^{2}-\left(1+k_{y} \sigma_{2}\right)^{2}\left|e_{y}\right|^{2} . \\
& e_{z}^{2} \leq e_{\theta}^{2}+2\left|e_{\theta}\right|\left|g\left(t, e_{y}\right)\right|+\left|g\left(t, e_{y}\right)\right|^{2} \leq 2 e_{\theta}^{2}+2\left(1+k_{y} \sigma_{2}\right)^{2}\left|e_{y}\right|^{2},
\end{aligned}
$$

we see that the following bounds on $V$ follow

$$
\begin{gathered}
V(t, e) \geq \frac{1}{2} \frac{c_{R}^{2}}{k_{\theta} k_{x}}\left[e_{x}^{2}+e_{y}^{2}\right]+\frac{1}{4} e_{\theta}^{2} \\
V(t, e) \leq\left[\frac{1}{2} \frac{c_{R}^{2}}{k_{\theta} k_{x}}+2\left(1+k_{y} \sigma_{2}\right)^{2}\right]\left[e_{x}^{2}+e_{y}^{2}\right]+e_{\theta}^{2} .
\end{gathered}
$$

Thus the inequalities in (65) also hold.

Condition c. Since the solutions are uniformly globally bounded, for any $r>0$, there exists $R>0$ such that $|e(t)| \leq R:=\{|e| \leq R\}$ for all $t \geq t_{\circ}$, all $e_{\circ} \in B_{r}$, and all $t_{\circ} \geq 0$. It is only left to establish uniform global attractivity. To that end, we observe that the nominal $\dot{e}=F(t, e)$ has the form

$$
\begin{aligned}
\dot{e}_{\theta} & =-k_{\theta} e_{\theta}-k_{y} f_{\rho}\left(t, e_{x}, e_{y}\right) \\
{\left[\begin{array}{c}
\dot{e}_{x} \\
\dot{e}_{y}
\end{array}\right] } & =\left[\begin{array}{cc}
-k_{x} & \Phi_{\theta}\left(t, e_{x}, e_{y}\right) \\
-\Phi_{\theta}\left(t, e_{x}, e_{y}\right) & 0
\end{array}\right]\left[\begin{array}{l}
e_{x} \\
e_{y}
\end{array}\right]
\end{aligned}
$$

where, for each $e_{\theta} \in B_{R}$, we define the smooth parameterized function $\Phi_{\theta}: \mathbb{R}_{\geq 0} \times \mathbb{R}^{2} \rightarrow$ $\mathbb{R}$ as

$$
\Phi_{\theta}\left(t, e_{x}, e_{y}\right):=\Phi\left(t, e_{\theta}, e_{x}, e_{y}\right)
$$

Then, the system (72) may be regarded as a cascaded system — cf. Loría (2008). Moreover, the system (72a) is input-to-state stable and the perturbation term $k_{y} f_{\rho}\left(t, e_{x}(t), e_{y}(t)\right)$ is uniformly bounded. Therefore, in order to apply a statement for cascaded systems, we must establish that the origin of $(72 \mathrm{~b})$ is globally asymptotically stable, uniformly in the initial conditions $\left(t_{\circ}, e_{x \circ}, e_{y \circ}\right) \in \mathbb{R}_{\geq 0} \times \mathbb{R}^{2}$ and in the "parameter" $e_{\theta} \in B_{R}$. For this, we invoke (Loría et al., 2002, Theorem 3) as follows. Since $k_{x}>0$ there is only left to show that $\Phi_{\theta}^{\circ}\left(t, e_{y}\right) e_{y}$, where

$$
\Phi_{\theta}^{\circ}\left(t, e_{y}\right):=\Phi_{\theta}\left(t, 0, e_{y}\right)
$$

is uniformly $\delta$-persistently exciting with respect to $e_{y}$, uniformly for any $\theta \in B_{R}-c f$. (Loría et al., 2002, Definition 3). Since $\Phi_{\theta}^{\circ}$ is smooth, it suffices to show that for any $\left|e_{y}\right| \neq 0$ and $r$, there exist $T$ and $\mu$ such that

$$
\left|e_{y}\right| \neq 0 \Longrightarrow \int_{t}^{t+T}\left|\tilde{\Phi}_{\theta}^{\circ}\left(\tau, e_{y}\right)\right| d \tau \geq \mu \quad \forall t \geq 0
$$

—see (Loría et al., 2002, Lemma 1). 
Remark 3. In general, $\mu$ depends both on $e_{\theta}$ and on $e_{y}$, but since $e_{\theta} \in B_{R}$ and $B_{R}$ is compact, by continuity, one can always choose the smallest qualifying $\mu$, for each fixed $e_{y}$. Therefore, as in Loría et al. (2002), $\mu$ may be chosen as a class $\mathcal{K}$ function dependent of $\left|e_{y}\right|$ only.

Now, we show that (73) holds under Assumption A2. To that end, we remark that

$$
\Phi_{\theta}^{\circ}\left(t, e_{y}\right)=k_{\theta} e_{\theta}+k_{y} \rho(t) f_{\circ}\left(t, e_{y}\right)
$$

$-c f$. Eq. (53), satisfies

$$
\dot{\Phi}_{\theta}^{\circ}=-k_{\theta} \Phi+k_{y} \dot{\rho} f_{\circ}+k_{y} \rho \frac{\partial f_{\circ}}{\partial t}-k_{y} \rho \frac{\partial f_{\circ}}{\partial e_{y}} \Phi e_{x}
$$

where we used $\dot{e}_{\theta}=-\Phi$ and $\dot{e}_{y}=\Phi e_{x}$. Therefore, defining

$$
K_{\Phi}(t, e):=k_{\theta}\left[\Phi_{\theta}^{\circ}-\Phi\right]-k_{y} \rho \frac{\partial f_{\circ}}{\partial e_{y}} \Phi e_{x}
$$

we obtain

$$
\dot{\Phi}_{\theta}^{\circ}=-k_{\theta} \Phi_{\theta}^{\circ}-k_{y} \rho \frac{\partial f_{\circ}}{\partial t}+k_{y} \dot{\rho} f_{\circ}+K_{\Phi}(t, e) .
$$

The latter equation corresponds to that of a linear filter with state $\Phi_{\theta}^{\circ}$ and input

$$
\Psi\left(t, e_{y}\right):=-k_{y} \rho(t) \frac{\partial f_{\circ}}{\partial t}\left(t, e_{y}\right)+k_{y} \dot{\rho}(t) f_{\circ}\left(t, e_{y}\right)+K_{\Phi}(t, e(t))
$$

therefore, $\Phi_{\theta}^{\circ}$ is uniformly $\delta$-PE with respect to $e_{y}$, if so is $\Psi$ (see e.g. Ioannou and Sun (1996)). Now, from Assumption A1 and uniform global boundedness of the solutions, for any $r$ there exists $c>0$ such that

$$
\left|k_{y} \dot{\rho}(t) f_{\circ}\left(t, e_{y}(t)\right)+K_{\Phi}(t, e(t))\right| \leq c(r)\left[\left|e_{x}(t)\right|+|\dot{\rho}(t)|\right]
$$

Therefore, uniform $\delta$-PE with respect to $e_{y}$ of $\Psi$ follows from Assumption $\mathbf{A} 2$ and the fact that $\dot{\rho}$ and $e_{x}$ are uniformly square integrable. That $\dot{\rho} \in \mathcal{L}_{2}$, with a bound uniform in the initial times, follows from (18) because $v_{r}, \omega_{r}$, and $\rho$ are bounded and $\left|v_{r}\right|+\left|\omega_{r}\right|$ is uniformly integrable. That $e_{x}$ is uniformly $\mathcal{L}_{2}$ follows from (57) - see Remark 2.

This concludes the proof of UGAS for the nominal system $\dot{e}=f_{e}(t, e)$ hence, Claim 2. is proved.

This completes the proof of Proposition 1.

\section{Simulations}

To illustrate our main theoretical results we performed some simulation tests under Simulink ${ }^{\mathrm{TM}}$ of Matlab ${ }^{\mathrm{TM}}$, according to the two scenarios described previously.

The robot's physical parameters are taken from Fukao et al. (2000):

$$
M=\left[\begin{array}{ll}
m_{1} & m_{2} \\
m_{2} & m_{1}
\end{array}\right], \quad C(\dot{z})=\left[\begin{array}{cc}
0 & c \omega \\
-c \omega & 0
\end{array}\right]
$$


with $m_{1}=0.6227, m_{2}=-0.2577, c=0.2025, r=0.15$, and $b=0.5$. We used the control law $(22)$ with $\sigma(\tilde{\nu})=\left[\tanh \left(\tilde{\nu}_{1}\right) \tanh \left(\tilde{\nu}_{2}\right)\right]^{\top}$.

For the purpose of the first scenario, we define the reference velocities $v_{r}$ and $\omega_{r}$ as periodic functions (hence persistently exciting) - see Figure 1. Such references generate a "staircase-shappe" path that is asymptotically followed by the vehicle see Figure 5, where we show the simulation results for two different values of the vehicle's initial conditions. The initial conditions for the reference vehicle are set to $\left[x_{r}(0), y_{r}(0), \theta_{r}(0)\right]=[0,0,0]$ and those for the adaptation law $(25)$ are set to $\hat{\Theta}(0)=$ $\left(\hat{m}_{1}, \hat{m}_{2}, \hat{c}\right)=(0,0,0)$.

The desired distance between the actual vehicle and the reference is obtained by setting the desired orientation offset to zero and defining $\left[d_{x_{r}}, d_{y_{r}}\right]:=[0,0]$. The control gains are set to $k_{x}=1, k_{y}=.2, k_{\theta}=0.1, k_{d}=20$, and $\gamma=1 \times 10^{-5}$. The function $f$ which verifies the assumptions $\mathbf{A} 1$ and $\mathbf{A} 2$ is defined as $f\left(t, e_{x}, e_{y}\right):=p(t)\left|e_{x y}\right|$ with $p(t)=50 \sin (0.5 t)+5$; we notice that both $p(t)$ and $\dot{p}$ are persistently exciting signals. Therefore, the conditions (14), (15) and (17) hold.

The tracking position errors are depicted in Figure 2 while in Figure 3 are showed the vehicle's and the reference velocities. The input torques at the wheels are depicted in Figure 4. To avoid graphical saturation we provide the curves only for the case in which the initial conditions are $x(0)=2, y(0)=1$ and $\theta(0)=0$.

For the stabilization scenario S2, we use exponentially-fast decaying reference trajectories - see Figure 6 and the control gains $k_{d}=30, k_{y}=1$. In Figure 10 we show the path followed by vehicle starting from two different points in the plane, as well as the reference path generated by the fictitious vehicle, which comes to a full stop. The tracking position and velocity errors are depicted in Figures 7 and 8 respectively, the input torques at the wheels are depicted in Figure 9 for the case in which the initial conditions of the vehicle are $x(0)=1, y(0)=1$ and $\theta(0)=0$.

The controller's performance may be compared, for instance, to that of the controllers in Do et al. (2004b); Lee et al. (2001).

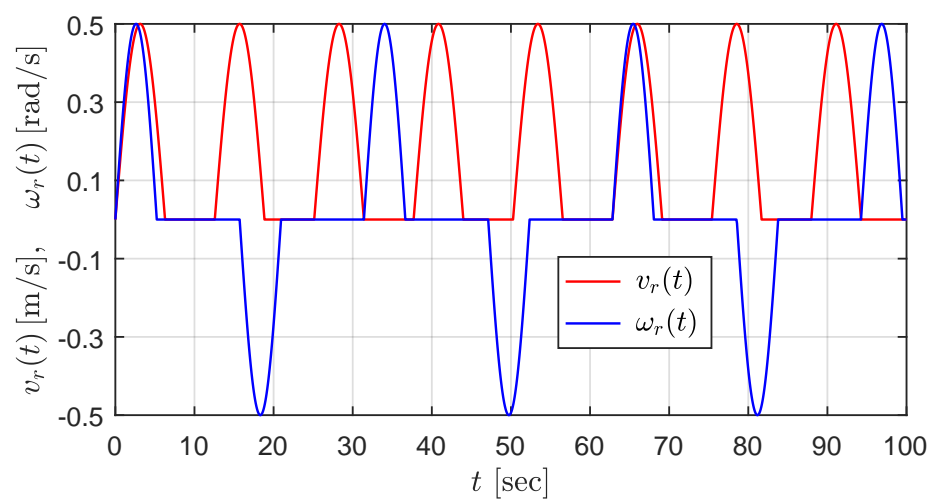

Figure 1. Persistently-exciting reference velocities $v_{r}$ and $\omega_{r}$ for the scenario $\mathbf{S 1}$

\section{Acknowledgements}

The authors are grateful to $\mathrm{H}$. Khalil for his keen technical remarks on the material presented in this paper. 


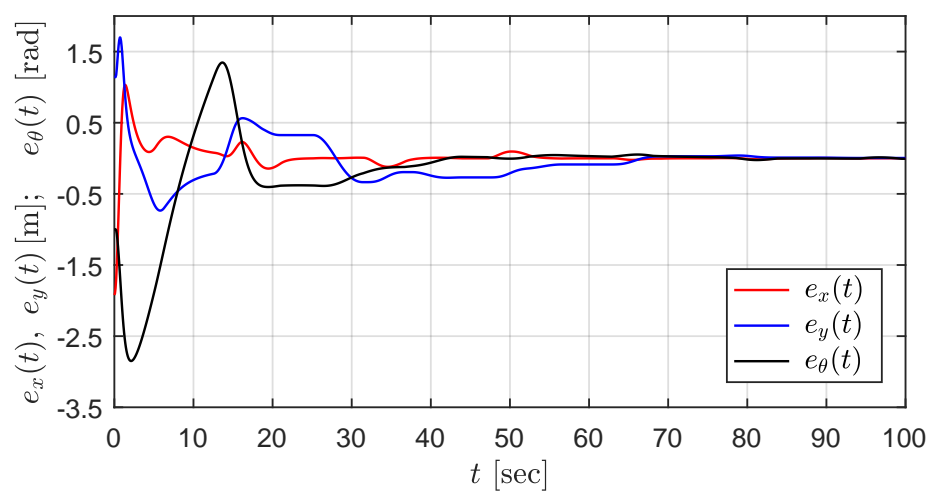

Figure 2. Tracking errors under the scenario S1
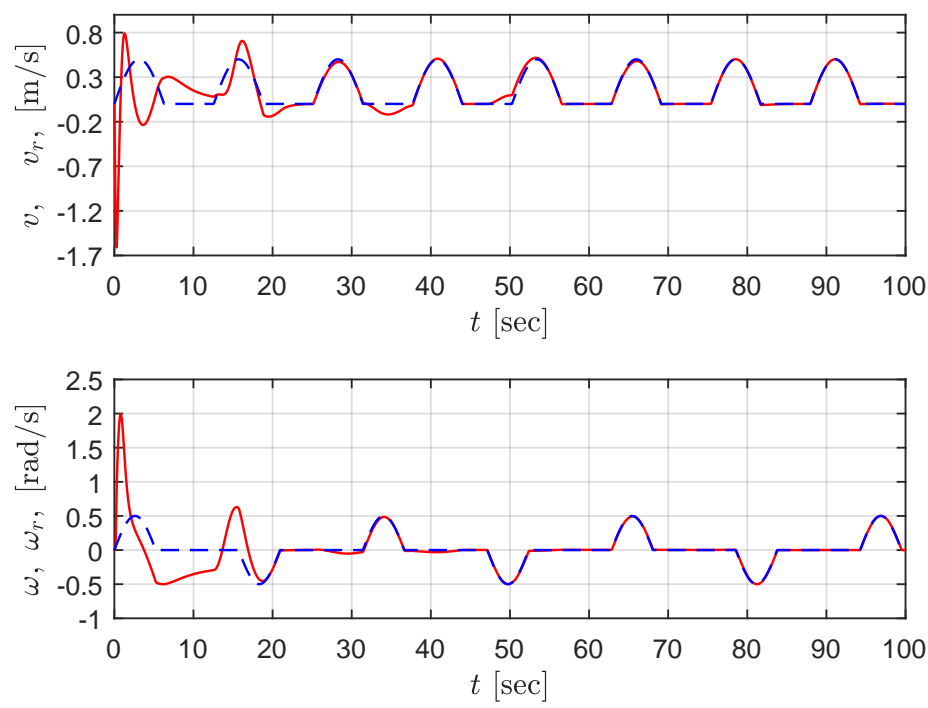

Figure 3. Vehicle's and reference velocities under the scenario S1

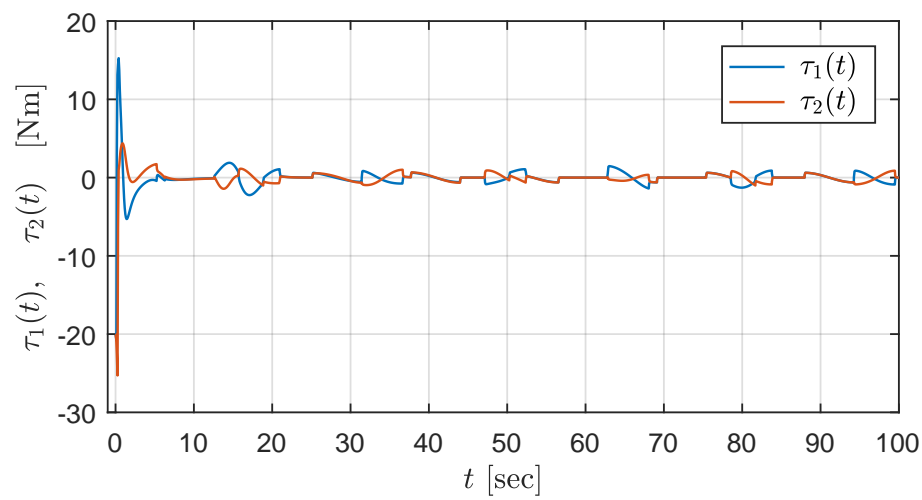

Figure 4. Input torques under the scenario $\mathbf{S 1}$

\section{Conclusion}

We presented a unique controller for nonholonomic vehicles with a generic dynamic model that achieves uniform global asymptotic stability in closed loop, for a large variety of reference trajectories. The simplicity and modularity of our design seems 


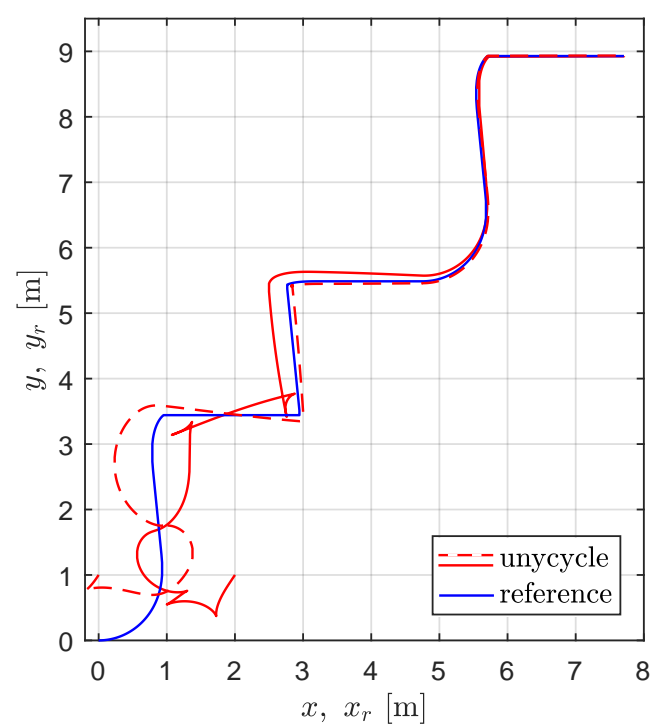

Figure 5. Path followed under the scenario S1 considering two different sets of initial conditions

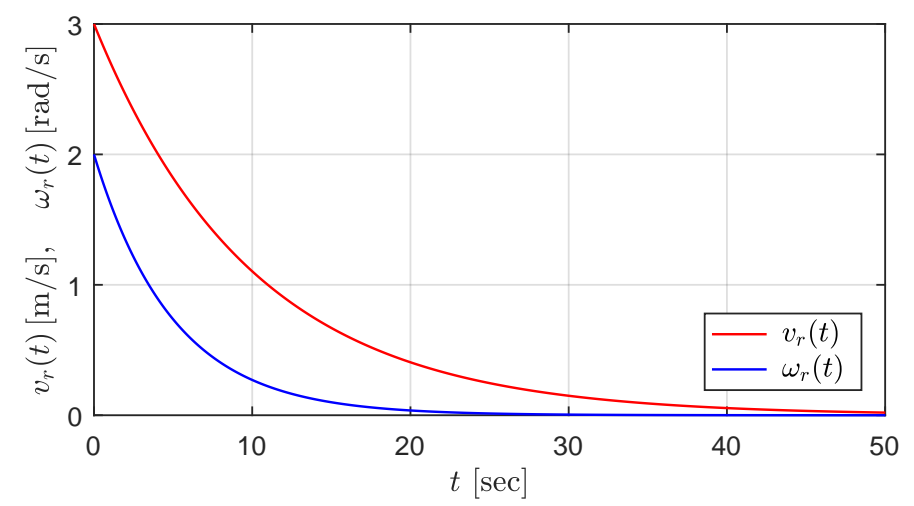

Figure 6. Exponentially-fast decaying reference velocities $v_{r}$ and $\omega_{r}$ for the scenario $\mathbf{S 2}$

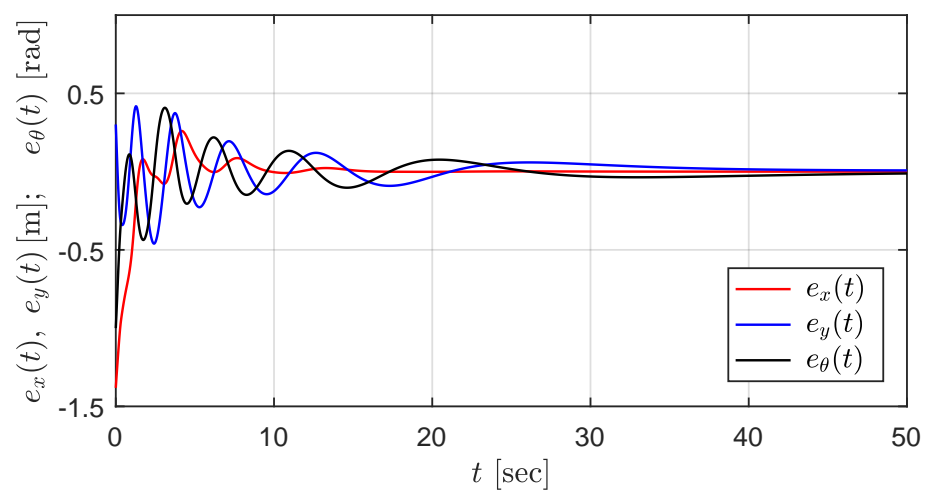

Figure 7. Tracking errors under the scenario S2

promising to broach other scenarios such as control under input constraints.

Our proofs are constructive for the tracking-control scenario; moreover, the construction of strict Lyapunov functions makes it possible to extend our designs to the cases of output feedback and parametric uncertainty. While an example of the latter is given, the former is under study. Furthermore, current research is being carried 

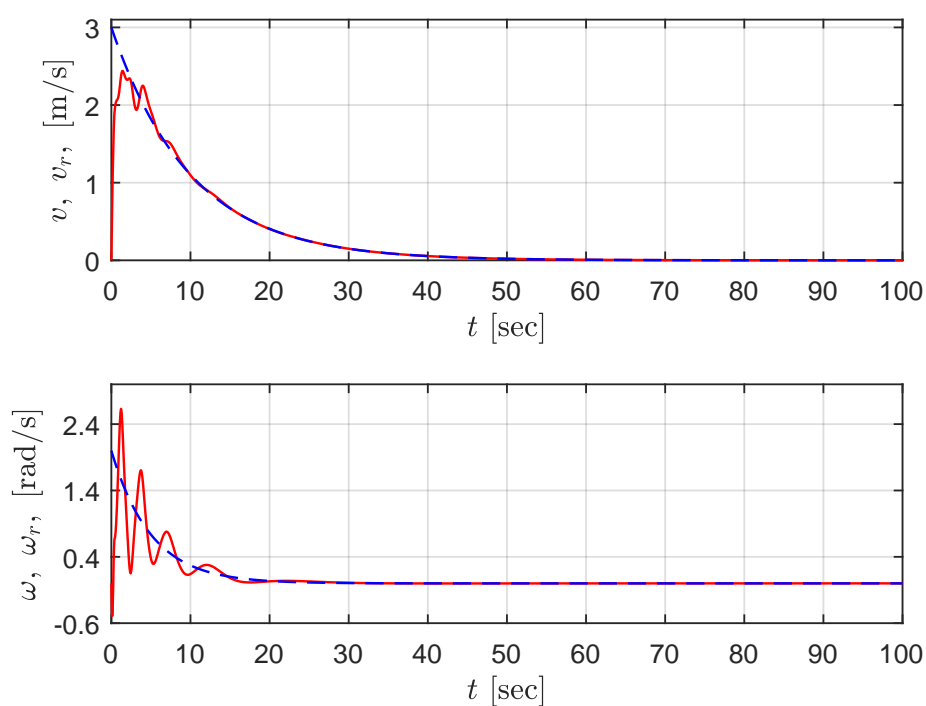

Figure 8. Vehicle and reference velocities under the scenario S2

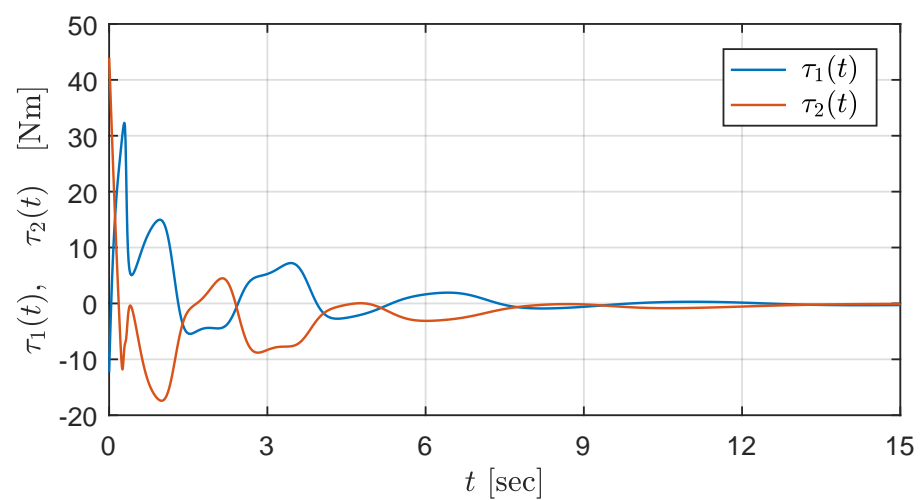

Figure 9. Input torques under the scenario S2

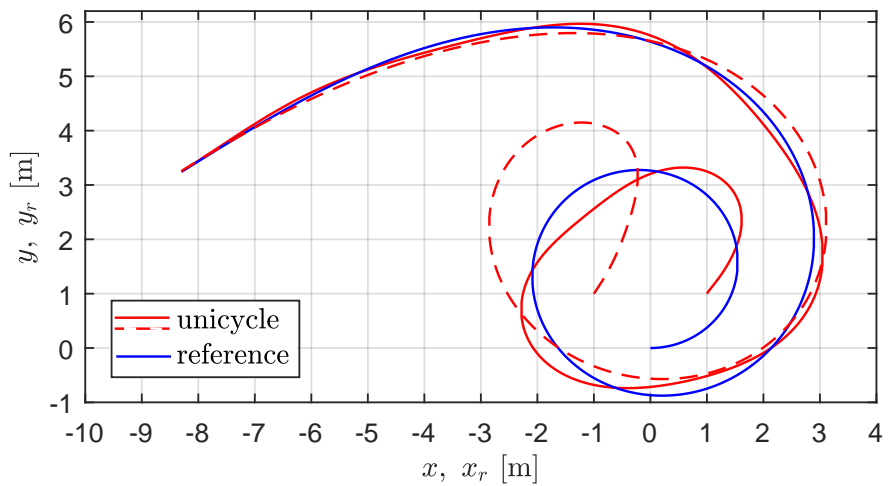

Figure 10. Path followed under the scenario S2 under different initial conditions

out to relax the standing assumption of integrability of the reference velocities in the stabilization scenario, to allow for slowly-converging reference velocities. 


\section{References}

Angeli, D., Ingalls, B., Sontag, E. D., \& Wang, Y. (2004). Separation principles for inputoutput and integral-input-to-state stability. SIAM J. on Contr. and Opt., 43(1), 256-276.

Angeli, D., Sontag, E. D., \& Wang, Y. (2000). A characterization of integral input-to-state stability. IEEE Trans. on Automat. Contr., 45(6), 1082-1097.

Astolfi, A. (1996). Discontinuous control of nonholonomic systems. Syst. \& Contr. Letters, $27(1), 37-45$.

Bayat, F., Mobayen, S., \& Javadi, S. (2016). Finite-time tracking control of nth-order chainedform non-holonomic systems in the presence of disturbances. ISA Transactions, 63, 78-83.

Brockett, R. (1983). Asymptotic stability and feedback stabilization. In R. S. M. R. W. Brocket \& H. J. Sussmann (Eds.), Differential geometric control theory (p. 181-191). Birkhäuser.

Cao, K.-C., \& Tian, Y.-P. (2007). A time-varying cascaded design for trajectory tracking control of non-holonomic systems. Int. J. of Control, 80(3), 416-429.

Consolini, L., Morbidi, F., Prattichizzo, D., \& Tosques, M. (2008). Leader-follower formation control of nonholonomic mobile robots with input constraints. Automatica, 44(5), 13431349.

de Wit, C. C., Khennouf, H., Samson, C., \& Sørdalen, O. J. (1993). "Nonlinear control design for mobile robots". In (Vol. 11, chap. Recent Trends in Mobile Robots). Y. F. Zheng, ed., London: World Scientific.

Dixon, W. E., Dawson, D. M., Zhang, F., \& Zergeroglu, E. (2000). Global exponential tracking control of a mobile robot system via a pe condition. IEEE Transactions Systems, Man, and Cybernetics B, 30(1), 129-142.

Dixon, W. E., Dawson, M. D., Zergeroglu, E., \& Behal, A. (2001). Nonlinear control of wheeled mobile robots. Springer Verlag. (ISBN 978-1-85233-414-7)

Dixon, W. E., de Queiroz, M. S., Dawson, D. M., \& Flynn, T. J. (2004). Adaptive tracking and regulation of a wheeled mobile robot with controller/update law modularity. IEEE Transactions on control systems technology, 12(1), 138-147.

Do, K. D. (2007, April). Formation tracking control of unicycle-type mobile robots. In Proceedings 2007 ieee international conference on robotics and automation (p. 2391-2396).

Do, K. D., Jiang, Z.-P., \& Pan, J. (2004a). A global output-feedback controller for simultaneous tracking and stabilization of unicycle-type mobile robots. IEEE Trans. on Robotics Automat., $20(3), 589-594$.

Do, K. D., Jiang, Z.-P., \& Pan, J. (2004b). Simultaneous tracking and stabilization of mobile robots: an adaptive approach. IEEE Trans. on Automat. Contr., 49(7), 1147-1152.

Fukao, T., Nakagawa, H., \& Adachi, N. (2000). Adaptive tracking control of a nonholonomic mobile robot. IEEE Trans. on Robotics Automat., 16(5), 609-615.

Hahn, W. (1967). Stability of motion. New York: Springer-Verlag.

Huang, J., Wen, C., Wang, W., \& Jiang, Z.-P. (2014). Adaptive output feedback tracking control of a nonholonomic mobile robot. Automatica, 50(3), 821-831.

Ioannou, P., \& Sun, J. (1996). Robust adaptive control. New Jersey, USA: Prentice Hall.

Jiang, Z.-P., Lefeber, E., \& Nijmeijer, H. (2001). Saturated stabilization and tracking of a nonholonomic mobile robot. Syst. \& Contr. Letters, 42(5), 327-332.

Jiang, Z.-P., \& Nijmeijer, H. (1997). Tracking control of mobile robots: A case study in backstepping. Automatica, 33(7), 1393-1399.

Kanayama, Y., Kimura, Y., Miyazaki, F., \& Naguchi, T. (1990). A stable tracking control scheme for an autonomous vehicle. In Proc. ieee conf. robotics automat. (p. 384-389).

Lee, T. C., Song, K. T., Lee, C. H., \& Teng, C. C. (2001, Mar). Tracking control of unicyclemodeled mobile robots using a saturation feedback controller. IEEE Trans. Contr. Syst. Technol., 9(2), 305-318.

Lefeber, A. A. J. (2000). Tracking control of nonlinear mechanical systems (Unpublished doctoral dissertation). University of Twente, Enschede, The Netherlands.

Lizárraga, D. A. (2004). Obstructions to the existence of universal stabilizers for smooth control systems. Mathematics of Control, Signals and Systems, 16, 255277. 
Loría, A. (2008). From feedback to cascade-interconnected systems: Breaking the loop. In Proc. 47th. ieee conf. decision contr. (p. 4109-4114). Cancun, Mex..

Loría, A., Dasdemir, J., \& Alvarez-Jarquin, N. (2016). Leader-follower formation and tracking control of mobile robots along straight paths. IEEE Trans. on Control Systems Technology, 24 (2), 727-732. (DOI: 10.1109/TCST.2015.2437328)

Loría, A., Panteley, E., Popovic, D., \& Teel, A. (2002). $\delta$-persistency of excitation: a necessary and sufficient condition for uniform attractivity. In Proc. 41th. ieee conf. decision contr. (pp. 3506-3511). Las Vegas, CA, USA. (Paper no. REG0623 )

Loría, A., Panteley, E., \& Teel, A. (1999). A new persistency-of-excitation condition for UGAS of NLTV systems: Application to stabilization of nonholonomic systems. In Proc. 5th. european contr. conf. (pp. 1363-1368). Karlsrühe, Germany.

Maghenem, M., Loría, A., \& Panteley, E. (2016). iISS formation tracking control of autonomous vehicles (Tech. Rep.). Gif sur Yvette, France: CentraleSupelec. (Available online: https://hal.archives-ouvertes.fr/hal-01364791)

Maghenem, M., Loría, A., \& Panteley, E. (2017). Global tracking-stabilization control of mobile robots with parametric uncertainty. In IFAC World Congress 201\%. Toulouse, France. (Accepted)

Malisoff, M., \& Mazenc, F. (2009). Constructions of strict Lyapunov functions. London: Springer Verlag.

Malkin, I. (1944). Ob ustořchivosti pri postoyanno deǐstvuyuschih vozmyscheniyah. Prikl. Mat. i Mekh., Tom. VIII, 241-245. (In Russian. Engl. translation: On stability under constantly acting disturbances)

Mazenc, F. (2003). Strict Lyapunov functions for time-varying systems. Automatica, 39 , 349-353.

Miao, Z., \& Wang, Y. (2015). Adaptive control for simultaneous stabilization and tracking of unicycle mobile robots. Asian. J. Contr., 17(6), 2277-2288. (DOI:10.1002/asjc.1142)

Morin, P., \& Samson, C. (2003). Practical stabilization of driftless systems on lie groups: the transverse function approach. IEEE Transactions on Automatic control, 48(9), 1496-1508.

Ortega, R., \& Spong, M. (1989). Adaptive motion control of rigid robots: A tutorial. Automatica, 25-6, 877-888.

Panteley, E., Lefeber, E., Loría, A., \& Nijmeijer, H. (1998). Exponential tracking of a mobile car using a cascaded approach. In Ifac workshop on motion control (p. 221-226). Grenoble, France.

Panteley, E., \& Loría, A. (2001). Growth rate conditions for stability of cascaded time-varying systems. Automatica, 37(3), 453-460.

Panteley, E., Loría, A., \& Teel, A. (2001). Relaxed persistency of excitation for uniform asymptotic stability. IEEE Trans. on Automat. Contr., 46(12), 1874-1886.

Samson, C. (1993). Time-varying feedback stabilization of car-like wheeled mobile robots. The International journal of robotics research, 12(1), 55-64.

Samson, C. (1995). Control of chained system: Application to path following and time-varying point stabilization of mobile robots. IEEE Trans. on Automat. Contr., 40(1), 64-77.

Wang, Y., Miao, Z., Zhong, H., \& Pan, Q. (2015, July). Simultaneous stabilization and tracking of nonholonomic mobile robots: A lyapunov-based approach. IEEE Transactions on Control Systems Technology, 23(4), 1440-1450. 